\title{
Transgenic mice overexpressing the extracellular domain of NCAM are impaired in working memory and cortical plasticity
}

\author{
Leann H. Brennaman ${ }^{1,{ }^{*}}$, Gaga Kochlamazashvili2,, ${ }^{2}$, Luminita Stoenica ${ }^{3}$, Randall J. \\ Nonneman ${ }^{4}$, Sheryl S. Moy ${ }^{4,5}$, Melitta Schachner ${ }^{3,6}$, Alexander Dityatev ${ }^{2,3,7, \dagger}$, and Patricia \\ F. Maness ${ }^{1,4, \dagger}$ \\ ${ }^{1}$ Department of Biochemistry and Biophysics, UNC Schizophrenia Research Center, University \\ of North Carolina School of Medicine, Chapel Hill, NC 27599 \\ 2 Department of Neuroscience and Brain Technologies, Italian Institute of Technology, 16163 \\ Genova, Italy \\ ${ }^{3}$ Zentrum für Molekulare Neurobiologie Hamburg, University Medical Center Hamburg- \\ Eppendorf, 20251 Hamburg, Germany \\ ${ }^{4}$ Carolina Institute for Developmental Disabilities, University of North Carolina School of \\ Medicine, Chapel Hill, NC 27599 \\ ${ }^{5}$ Department of Psychiatry, University of North Carolina School of Medicine, Chapel Hill, NC \\ 27599 \\ ${ }^{6}$ Keck Center for Collaborative Neuroscience and Department of Cell Biology and Neuroscience, \\ Rutgers University, Piscataway, NJ 08854, USA \\ 7 Institut für Neurophysiologie und Pathophysiologie, University Medical Center Hamburg- \\ Eppendorf, 20246 Hamburg, Germany
}

\begin{abstract}
The neural cell adhesion molecule, NCAM, is a pivotal regulator of neural development, with key roles in axonal and dendritic growth and synaptic plasticity. Alterations in NCAM expression or proteolytic cleavage have been linked to human neuropsychiatric disorders such as schizophrenia, bipolar disorder and Alzheimer's disease, and may contribute to cognitive dysfunction. We have generated mice overexpressing the NCAM extracellular (EC) proteolytic cleavage fragment which has been reported to be increased in schizophrenic versus normal brains. These mice show impaired GABAergic innervation and reduced number of apical dendritic spines on pyramidal neurons in the prefrontal cortex (PFC). Here, these NCAM-EC transgenic mice were subjected to behavioral tasks and electrophysiological measurements to determine the impact of structural abnormalities in the PFC on synaptic and cognitive functions. NCAM-EC mice exhibited impaired working memory in a delayed non-match-to-sample task, which requires PFC function, but showed no differences in anxiety, olfactory abilities, or sociability. Transgenic mice displayed impaired long- and short-term potentiation in the PFC but normal synaptic plasticity in the hippocampus, suggesting that the abnormal synaptic innervation in NCAM-EC mice impairs PFC
\end{abstract}

() 2011 Elsevier Inc. All rights reserved.

${ }^{\dagger}$ To whom correspondence should be addressed: Dr. Alexander Dityatev, +39-010-71781515 (phone), +39-010-71781230 (fax), alexander.dityatev@ iit.it or Dr. Patricia F. Maness, +1-919-966-3532 (phone), +1 919-966-2852 (fax), srclab@med.unc.edu. These authors contributed equally to this work.

Publisher's Disclaimer: This is a PDF file of an unedited manuscript that has been accepted for publication. As a service to our customers we are providing this early version of the manuscript. The manuscript will undergo copyediting, typesetting, and review of the resulting proof before it is published in its final citable form. Please note that during the production process errors may be discovered which could affect the content, and all legal disclaimers that apply to the journal pertain. 
plasticity and alters working memory. These findings may have implications for cognitive dysfunctions observed in neuropsychiatric disorders.

\section{Keywords}

prefrontal cortex; NCAM; working memory; synaptic plasticity; neuropsychiatric disorders; LTP; STP

\section{Introduction}

Cell adhesion molecules are now accepted not only to be important for development but also for synaptic plasticity (Arikkath and Reichardt, 2008; Dalva et al., 2007; Dityatev et al., 2008; Gottmann, 2008; Schachner, 1997). Among the adhesion molecules, the neural cell adhesion molecule, NCAM, was the first to be shown to affect long-term potentiation (LTP) and behavior (Becker et al., 1996; Lüthi et al., 1994; Muller et al., 1996). NCAM is a cell adhesion molecule of the immunoglobulin superfamily that plays key roles in neural development, shaping axonal and dendritic arborizations. Polymorphisms in the NCAM gene have also been implicated in a variety of neuropsychiatric disorders (reviewed by (Brennaman and Maness, 2010), underscoring its importance in normal physiology and/or development. Three major isoforms of NCAM (NCAM 180, 140, 120) are derived by alternative splicing. NCAM140 and NCAM180 are transmembrane isoforms that differ in the length of the cytoplasmic domain due to an inserted exon in NCAM180. NCAM120 is glycosylphosphatidylinositol-linked and predominantly found in glia. NCAM participates in NCAM-NCAM homophilic interactions, and heterophilic interactions with binding partners such as cell adhesion molecule L1 (Kadmon et al., 1990), heparin sulfate proteoglycans (Storms and Rutishauser, 1998), fibroblast growth factor receptor (FGFR) (Niethammer et al., 2002), the brain-derived neurotrophic factor (BDNF) receptor TrkB (Cassens et al., 2010), and glial derived neurotrophic factor (GDNF) and its receptor (Paratcha et al., 2003) (Nielsen et al., 2009). Mice lacking all NCAM isoforms exhibit impaired learning (Cremer et al., 1994; Stork et al., 2000) and fear conditioning (Jurgenson et al., 2010; Senkov et al., 2006), as well as increased inter-male aggression, anxiety, locomotion, and depression-like symptoms (Polo-Parada et al., 2001; Stork et al., 1997; Stork et al., 1999). NCAM-null mice also display impaired synaptic plasticity as measured by hippocampal LTP in vitro (Kochlamazashvili et al., 2010; Muller et al., 1996) and in vivo (Stoenica et al., 2006).

NCAM is subject to post-translational modifications that alter its functions. NCAM is glycosylated in its extracellular domain by polysialyltransferases, ST8SiaII and ST8SiaIV, which add long chains of polysialic acid (PSA) to the NCAM glycoprotein backbone (Hildebrandt et al., 2007). PSA-NCAM is expressed from embryonic stages through early postnatal development and remains expressed in regions of the mature brain with high synaptic plasticity, such as the hippocampus. The presence of PSA reduces NCAM-NCAM homophilic and also heterophilic binding, which promotes axon/dendrite arborization in early development or facilitates synaptogenesis and synaptic plasticity at later stages (reviewed by (Bonfanti, 2006). The latter involves inhibition of extrasynaptic GluN2Bcontaining NMDA receptors by PSA (Kochlamazashvili et al., 2010).

Another post-translational modification of NCAM is proteolytic cleavage that leads to a soluble NCAM extracellular domain fragment (NCAM-EC) (Hinkle et al., 2006; Kalus et al., 2006). NCAM-EC is expressed during normal human and mouse neural development (Brennaman and Maness, 2008; Cox et al., 2009), but its levels are upregulated in neuropsychiatric disorders such as schizophrenia, bipolar disorder and Alzheimer's disease (reviewed in (Brennaman and Maness, 2010). A transgenic mouse that overexpresses 
NCAM-EC (Pillai-Nair et al., 2005) at levels similar to those observed pathologically (Vawter, 2000) exhibits decreased numbers of inhibitory synapses and dendritic spines in the prefrontal cortex (PFC) (Pillai-Nair et al., 2005). These transgenic mice display a decline in development of perisomatic innervation from basket cells in the PFC (Brennaman and Maness, 2008; Pillai-Nair et al., 2005), which may be involved in synchronizing firing of pyramidal cells important for cognitive functions, such as working memory (Klausberger et al., 2003; Sohal et al., 2009). NCAM-EC mice also display impaired fear conditioning, decreased pre-pulse inhibition of acoustic startle reflex, and increased locomotion when compared to normal, wild type (WT) control littermates on the C57BL/6 background (PillaiNair et al., 2005).

Here we examined whether synaptic alterations observed in the PFC of NCAM-EC mice are manifested in behavioral or synaptic abnormalities. Mice were examined for deficits in a delayed non-match-to-sample working memory task, a behavioral paradigm that is a wellestablished model of PFC function (Kellendonk et al., 2006), and for deficits in prefrontal and hippocampal synaptic transmission and plasticity. NCAM-EC mice were found to have defective working memory and impaired LTP and short-term potentiation (STP) in the PFC.

\section{Materials and methods}

\section{Mice}

Separate sets of young adult inbred C57BL/6 NCAM-EC transgenic mice and their WT littermates of both sexes were used for the electrophysiological experiments (2-3 months in age) and behavioral measurements (2-6 months in age). All treatments of animals were approved by the University of North Carolina Animal Care and Use Committee and the Committees on Animal Health and Care of the governmental bodies in Hamburg and Rome in accordance with NIH guidelines.

\section{Behavioral testing procedures}

Two separate sets of mice were used in the behavioral studies. The first set of mice consisted of $10 \mathrm{WT}$ and 10 NCAM-EC mice (with 5 males and 5 females in each group), around 3 months of age at the start of testing. This set was evaluated for working memory function in the delayed non-match to sample task. The second set of mice included 10 WT (3 male and 7 female) mice and 9 NCAM-EC ( 5 male and 4 female) mice, around 2 months of age at the start of testing. These mice were evaluated with the following assays, in the given order: 1) open field, 2) olfactory test with no food deprivation, 3) three-chambered social approach test, 4) olfactory test with food deprivation, and 5) elevated plus maze for anxiety-like behavior.

\section{Delayed non-match to sample (DNMTS) test}

Mice were group housed and food deprived to $80-85 \%$ of their ad libitum starting weight before beginning the DNTMS test, which was based on the protocol described by Kellendonk and colleagues (Kellendonk et al., 2006). Briefly, mice were habituated to the Tmaze by allowing them to collect a malted milk solution $(0.2 \mathrm{ml}$, Nestle Carnation Malted Milk Powder) from each arm of the maze over 3 days. Mice were then given 4 trials per day over a 15 day period, with each trial consisting of a forced choice and a free choice. For the forced choice, mice were placed in a start box at the end of the T-maze stem and then allowed to travel up the stem and enter one arm of the maze to collect a malted milk reward. The opposite arm was closed with a door across the entrance. Mice were then placed back in the start box for the free choice run. In this case, both arms were open, but only the arm that had been previously closed contained a reinforcer. After a $4 \mathrm{~s}$ delay, mice were given a choice between the two arms. A correct choice was scored if the mouse chose the opposite 
arm from the open arm in the forced choice run. For both the forced choice and free choice runs, a door was used to prevent mice from leaving an arm after entry. Trials were separated by at least $20 \mathrm{~min}$. Following the first 15 -day test with a minimal $4 \mathrm{~s}$ delay period, mice were run for an additional 16 days with a $15 \mathrm{~s}$ delay between the forced choice run and the free choice run. Criterion for learning was set at 11 out of 12 correct choices over 3 days, according to the methodology of Kellendonk and colleagues (Kellendonk et al., 2006). The maze was cleaned with water and dried with paper towels between each animal, and wiped with ethanol between female and male groups.

\section{Open field and elevated plus maze tests}

These tests are standard measures of anxiety-like behavior in rodents. The procedures are based on the natural tendency of mice to actively explore a new place, versus a fear of being in an open area. For the open field test, each mouse was given one 5-min trial in an acrylic locomotor chamber $(40 \mathrm{~cm} \times 40 \mathrm{~cm} \times 30 \mathrm{~cm})$, and counts were taken of the number of photobeams broken during the trial (VersaMax system, AccuScan Instruments, Columbus, $\mathrm{OH})$. Measures included locomotor activity (total distance traveled), rearing movements, and time spent in the center region. For the elevated plus maze test, each mouse was given one 5-min trial on a maze with two walled arms (the closed arms, $40 \mathrm{~cm}$ in height) and two open arms. The maze was elevated $50 \mathrm{~cm}$ from the floor, and the arms were $21 \mathrm{~cm}$ long. Animals were placed on the center section $(9.5 \mathrm{~cm} \times 9.5 \mathrm{~cm})$, and allowed to freely explore the maze. Measures were taken of time on, and number of entries into, the open and closed arms. Total number of entries on the elevated plus maze was used as an index of overall activity in the assay.

\section{Buried food test for olfactory function}

Mice received two buried food tests to assess olfactory ability. Two and three days before each olfactory test, pieces of cereal (Froot Loops, Kellogg Co., Battle Creek, MI) were placed overnight in the home cages to familiarize the mice to the food. On the day of the first test, each mouse was placed in a large, clean tub cage $(46 \mathrm{~cm} \mathrm{~L} \mathrm{x} 23.5 \mathrm{~cm} \mathrm{~W}$ x $20 \mathrm{~cm}$ $\mathrm{H}$ ), containing $3 \mathrm{~cm}$ deep paper chip bedding, and allowed to explore for $5 \mathrm{~min}$. The animal was removed from the cage, and one Froot Loop was buried in the cage bedding. The animal was then returned to the cage and given 15 min to locate the buried food. Measures were taken of latency to find the food reward and whether it was consumed. The second olfactory test was conducted using the same procedure, except that mice were food-deprived for 1620 hours before the start of the test.

\section{Three-chambered social approach test}

The social approach test consisted of three 10 min phases: a habituation period, a test for sociability, and a test for social novelty (Moy et al., 2004; Nadler et al., 2004). For the sociability assay, mice were given a choice between being in the proximity of an unfamiliar conspecific versus being alone. In the social novelty test, the mice were given a further choice between spending time with the first unfamiliar mouse, or with a newly introduced animal. The social testing apparatus was a rectangular, three-chambered box fabricated from clear polycarbonate (described in Nadler et al., 2004). Dividing walls had doorways allowing access into each chamber. Photocells were embedded in each doorway to allow automatic quantification of entries and duration in each side of the social test box. The chambers of the apparatus were cleaned between each trial.

\section{Sociability test}

The test mouse was first placed in the middle chamber and allowed to explore for $10 \mathrm{~min}$, with the doorways into the two side chambers open. After the habituation period, the test 
mouse was enclosed in the center compartment of the social test box, and an unfamiliar C57BL/6J male (stranger 1) was placed in one of the side chambers. The stranger mouse was enclosed in a small wire cage which allowed nose contact between bars, but prevented fighting. An identical empty wire cage was placed in the opposite side of the test box, after which the doors were re-opened and the subject allowed to explore the entire social test box for $10 \mathrm{~min}$. Measures were taken of the amount of time spent in each chamber and the number of entries into each chamber. In addition, records were taken for time spent sniffing each wire cage by a human observer seated 5 feet from the apparatus.

\section{Social novelty preference test}

At the end of the first $10 \mathrm{~min}$, each mouse was tested in a second $10 \mathrm{~min}$ session to quantitate social novelty preference for a new stranger. A second unfamiliar mouse was placed in the wire cage that had been empty in the previous session. The test mouse had a choice between the first, already-investigated unfamiliar mouse (stranger 1), and the novel unfamiliar mouse (stranger 2). As described above, measures were taken of the amount of time spent in each chamber, the number of transitions between chambers of the apparatus, and time spent sniffing each wire cage.

\section{Electrophysiological recordings}

After brief $\mathrm{CO}_{2}$ sedation, decapitation, and removal of the brain, transverse $350 \mu \mathrm{m}$-thick hippocampal slices or coronal $400 \mu \mathrm{m}$-thick cortical slices containing the prefrontal area were cut with a VT1200M vibratome (Leica, Nussloch, Germany) in ice-cold artificial cerebrospinal fluid (ACSF) infused with $95 \% \mathrm{O}_{2} / 5 \% \mathrm{CO}_{2}$ and containing (in $\mathrm{mM}$ ): 250 sucrose, $24 \mathrm{NaHCO}_{3}, 25$ glucose, $2.5 \mathrm{KCl}, 1.25 \mathrm{NaH}_{2} \mathrm{PO}_{4}, 2 \mathrm{CaCl}_{2}, 1,5 \mathrm{MgSO}_{4}$ (pH 7.4). The slices were then kept for at least two hours before the start of recordings in carbogeninfused ACSF, containing $120 \mathrm{mM} \mathrm{NaCl}$ instead of sucrose (Eckhardt et al., 2000). Recordings were performed in the same solution, in a submerged chamber, at room temperature $\left(22-24^{\circ} \mathrm{C}\right)$. Recordings of field excitatory postsynaptic potential (fEPSP) in the hippocampus were performed in the stratum radiatum of the CA1b subfield with glass pipettes filled with artificial cerebrospinal fluid (ACSF) and having a resistance of 1-2 M 2 . Schaffer collaterals were stimulated with a $1 \mathrm{M} \Omega$ - glass electrode placed approximately 300 $\mu \mathrm{m}$ closer to the CA3 subfield than the recording electrode. Recordings in the medial PFC were performed with the same types of glass electrodes. A stimulation electrode was placed in the layers II-III of the PFC and recordings were taken from layer V, where the basal dendrites and cell bodies of pyramidal neurons are located (Huang et al., 2004).

Basal synaptic transmission and paired-pulse facilitation were studied for both types of synapses before inducing LTP. Stimulus-response curves were constructed as a measure of basal level of synaptic transmission. Paired-pulse facilitation at 10, 20, 50, 100 and $200 \mathrm{~ms}$ interpulse intervals was studied as a measure of short-term plasticity. The stimulation intensity in these experiments was set to elicit fEPSPs with magnitude of approximately $30 \%$ from the maximum (defined as a response with detectable population spike). Basal synaptic transmission was monitored at $0.05 \mathrm{~Hz}$. The inter-theta-train interval was $20 \mathrm{~s}$ and three trains were applied to induce LTP in the CA1 while five trains were used in the PFC. Each train consisted of 8 bursts delivered at $5 \mathrm{~Hz}$. Each burst consisted of four pulses delivered at $100 \mathrm{~Hz}$. Duration of pulses was $0.2 \mathrm{~ms}$ and stimulation intensity was set to provide baseline fEPSPs with magnitudes of approximately $50 \%$ from the maximum. The signals were recorded using the EPC10 amplifier at sampling rate of $10 \mathrm{kHz}$, low-pass filtered at $3 \mathrm{kHz}$ and analyzed using PatchMaster software (both from Heka Elektronik, Lambrecht/Pfalz, Germany). SigmaPlot (Systat Software Inc., Chicago, IL) was used for data presentation and digital subtraction of responses elicited by single pulses for precise measurements of paired-pulse facilitation (defined as the ratio between the slopes of fEPSPS 
elicited by the second and first pulses). The level of short-term potentiation was determined as the maximal potentiation within first $2 \mathrm{~min}$ after theta-burst stimulation. The measurements of parameters were performed blindly, i.e. without knowing the genotypes of recorded mice. The stimulus artifacts were blanked to facilitate perception of fEPSPs.

\section{Statistical analysis}

Electrophysiological and behavioral data were analyzed using t-tests or repeated measures ANOVAs (analysis of variance), with the factor genotype. Sex was not included as a factor in the analyses, because the sample sizes did not confer enough power to detect both genotype and sex effects. Significant main effects or interactions were further explored using post-hoc Fisher's PLSD (protected least-significant difference) tests to compare group means. Social preference was determined using within-genotype repeated measures ANOVAs, with the factor of chamber side (e.g., stranger 1 side or the opposite side). For all comparisons, significance was set at $\mathrm{p}<0.05$.

\section{Results}

\section{Impaired working memory but normal sociability, olfactory function, and anxiety responses in NCAM-EC mice}

Working memory in rodents using a delayed-non-match to sample (DNMTS) T-maze task requires an intact PFC (Kellendonk et al., 2006). We previously demonstrated that NCAMEC transgenic mice displayed impaired perisomatic GABAergic synaptic development in the PFC (Brennaman and Maness, 2008; Pillai-Nair et al., 2005). Since perisomatic innervation synchronizes pyramidal cell groups to promote working memory (Klausberger et al., 2003; Sohal et al., 2009), WT and NCAM-EC transgenic mice were tested in a DNMTS T-maze task with either a 4 or $15 \mathrm{~s}$ delay to determine if the disrupted inhibitory synapses in the PFC of NCAM-EC mice may alter working memory. In the $4 \mathrm{~s}$ delay, $90 \%$ of WT mice and only 50\% of NCAM-EC mice met criterion (Fig. 1A; criterion is 11 of 12 correct choices over 3 days (Kellendonk et al., 2006)). The same cohort of mice was retested with a $15 \mathrm{~s}$ delay to determine if a longer delay would result in further impairment. The impaired working memory in the NCAM-EC mice persisted (Fig. 1B) with 70\% of NCAMEC and $80 \%$ of WT meeting criterion over 16 days. The average days to criterion ( \pm standard error of the mean) for each group were almost identical with either the $3-4 \mathrm{~s}$ or $15 \mathrm{~s}$ delay: $8.6 \pm 1.3$ and $8.5 \pm 1.5$ for WT and $12.3 \pm 1.0$ and $12.4 \pm 1.1$ for NCAM-EC, respectively. Although more NCAM-EC mice met criterion with the $15 \mathrm{~s}$ delay, the mutants showed a significantly higher error rate than the WT mice in this phase of testing (Figure S1). Repeated measures ANOVA, conducted on the percent correct trials per day (Figure S1), showed that the impairment of the NCAM-EC mice closely approached significance at the $4 \mathrm{~s}$ delay $[\mathrm{F}(1,18)=4.35, \mathrm{p}=0.0516]$ and reached significance with the longer delay $[F(1,18)=6.28, p=0.0220]$. A repeated measures ANOVA with the factors genotype and delay, indicates a significant effect of genotype on days to criterion $[F(1,18)=8.16$, $\mathrm{p}=0.0105]$, but no effect of genotype by delay interaction $[\mathrm{F}(1,18)=0.01, \mathrm{p}=0.934]$. This analysis confirms that the groups had similar performance during the two phases of testing, and that NCAM-EC mice were impaired in the DNMTS task.

Possible factors underlying performance deficits in the T-maze task could be altered exploration in a novel environment and increased anxiety-like behavior in NCAM-EC transgenic mice. A separate group of WT and NCAM-EC mice were evaluated in open field and elevated plus maze tasks. There were no differences between the two groups for distance traveled, rearing movements, or time spent in the center region of the open field (Table 1). In the elevated plus maze, both WT and NCAM-EC mice spent a similar percentage of time in the open arms and had similar total numbers of arm entries (Table 1), 
suggesting that altered NCAM-EC function does not lead to changes in anxiety or activity in novel environments. As another control, mice were tested in a buried food assay to determine if a lack of olfactory ability contributed to the NCAM-EC working memory deficit. Mice were presented with an unfamiliar food (Froot Loops) 2-3 days prior to testing. On the day of testing, a Froot Loop was buried in a clean cage and mice were given the opportunity to find and consume it. No differences were observed in WT or NCAM-EC mice in latency to uncover the buried food (Table $1 ; \mathrm{t}(17)=1.37, \mathrm{p}=0.189$ ), although high variability within the groups made the detection of significant differences difficult. A 16-20 hour period of food deprivation led to lower latencies to find the buried food in both groups of mice $[\mathrm{t}(17)=0.55, \mathrm{p}=0.5872]$. Taken together, these data indicate that the working memory deficit in the NCAM-EC transgenic mice is not due to increased anxiety or impaired olfactory ability.

In humans, damage to the PFC affects not only cognitive functions (Gehring and Knight, 2002; Manes et al., 2002), but also social and emotional function (Anderson et al., 2009; Bramham et al., 2009). To determine if NCAM-EC transgenic mice displayed altered social functions, WT and NCAM-EC mice were tested for sociability and social novelty. During the test for sociability, both the WT mice $[\mathrm{F}(1,9)=11.84, \mathrm{p}=0.0074]$ and the NCAM-EC animals $[\mathrm{F}(1,8)=26.77, \mathrm{p}=0.0008]$ demonstrated a clear preference, for spending more time in the side of the social test box containing the unfamiliar mouse, in comparison to the side with the empty wire cage (Fig. 2). During the social novelty assay, both groups of mice showed a switch in preference from the first unfamiliar mouse, stranger 1, to the newlyintroduced stranger 2 [WT, $F(1,9)=13.31$, $\mathrm{p}=0.0053$; NCAM-EC, $\mathrm{F}(1,8)=12.63$, $\mathrm{p}=0.0075$ ]. Repeated measures ANOVA did not reveal any significant effects of genotype on either sociability or social novelty. In addition, no effects of genotype were observed for measures of time spent sniffing each wire cage or entries into each chamber (data not shown). Thus, NCAM-EC mice showed normal social functions.

\section{Impaired synaptic plasticity in prefrontal cortex of NCAM-EC mice}

To search for physiological correlates of impaired working memory in NCAM-EC mice, we investigated basal synaptic transmission and synaptic plasticity in brain slice preparations from the hippocampus and medial PFC, two regions that are implicated in working memory (Brady et al., 2010; Finn et al., 2010).

To investigate effects of NCAM-EC overexpression on basal excitatory synaptic transmission, stimulus-response curves were obtained for field excitatory postsynaptic potentials (fEPSPs) in layer 5 of the medial PFC. No differences were found between NCAM-EC and WT mice (two-way ANOVA with repeated measures; Fig. 3A), nor were any differences detected in the paired-pulse ratio, a measure of short-term plasticity, at different interpulse intervals (two-way ANOVA with repeated measures; Fig. 3B). We next asked whether potentiation of fEPSPs induced by a repetitive theta-burst stimulation and measured $2 \mathrm{~min}$ (STP) or 50-60 $\mathrm{min}$ (LTP) after the stimulation would be influenced by NCAM-EC overexpression, as reported for hippocampal LTP in NCAM- and PSA-deficient mice (Eckhardt et al., 2000; Muller et al., 1996). The levels of STP were significantly reduced in NCAM-EC mice $(153.0 \pm 9.3 \%)$ compared to WT mice $(200.6 \pm 15.7 \%, \mathrm{p}=$ 0.024, unpaired t-test, Fig. 3C). Furthermore, the levels of LTP in NCAM-EC mice (110.3 \pm $2.9 \%)$ were significantly smaller in NCAM-EC than in WT mice $(126.7 \pm 4.2 \%, \mathrm{p}=0.007$, unpaired t-test; Fig. 3C).

Recordings of fEPSPs elicited by stimulation of Schaffer collaterals/commissural fibers and recorded in the stratum radiatum of the CA1 region of the hippocampus did not reveal any difference in stimulus-response curves or paired-pulse facilitation between the genotypes (two-way ANOVA Fig. 4A,B), the levels of STP $(248.9 \pm 17.2 \%$ in NCAM-EC mice and 
$218.2 \pm 9.8 \%$ in WT mice; $\mathrm{p}=0.112$, unpaired t-test; Fig. 4C) and the levels of LTP (145.4 $\pm 6.7 \%$ in NCAM-EC and $145.6 \pm 4.6 \%$ in WT; $p=0.95$, unpaired t-test; Fig. $4 \mathrm{C}$ ). Thus, electrophysiological recordings in slice preparations from NCAM-EC mice revealed selective impairment in synaptic plasticity in the PFC compared to hippocampus in response to repetitive theta-burst stimulation.

\section{Discussion}

Alterations in neuronal connectivity can have profound effects on the functional output of the synapse, from impaired or aberrant neurotransmission to cognitive dysfunction, such as impediments to learning and memory as well as social functions. Here we examined NCAM-EC transgenic mice for abnormalities in working memory and synaptic plasticity. NCAM-EC transgenic mice demonstrated impaired working memory in the DNMTS task. The NCAM-EC transgenic mice were impaired in the task at 4 and $15 \mathrm{~s}$. More NCAM-EC mutant mice reached criterion with the $15 \mathrm{~s}$ delay, but demonstrated a significantly higher error rate in correct choices per day than WT mice (Figure S1). Statistical analysis only detected an effect of genotype, but not delay, which implicates the elevated levels of NCAM-EC in the transgenic mice in impaired working memory in the DNMTS task. A longer, more challenging delay may also be necessary to demonstrate further impairment in the mutant mice. These studies focused on the effect of the NCAM-EC protein on working memory in the DNMTS task, using male and female mice of each genotype as a combined group. Statistical analysis lacked power to detect differences based on gender and genotype based on sample size. Other mouse models of schizophrenia (transmembrane domain mutation in Neuregulin-1 (Duffy, 2010), 22q11 deletion (Stark et al., 2008), and DISC1 mutants (Clapcote et al., 2007; Kellendonk et al., 2009) did not show a gender bias in working memory tasks, but only in anxiety, fear conditioning and locomotor tasks. Some studies reported a slightly higher prevalence of schizophrenia in men (Hovatta et al., 1997; Myles-Worsley et al., 1999), and a later onset of disease in women (Hafner and an der Heiden, 1997; Hafner et al., 1998; Myles-Worsley et al., 1999). On the other hand, based on combined prevalence estimates from 188 studies, no significant difference between males and females was detected (Saha et al., 2005). Core symptoms, including cognitive dysfunctions such as working memory deficits, have not shown a strong gender bias (AlUzri et al., 2006; Hafner and an der Heiden, 1997; Mohamed et al., 1999). Thus, we combined the mice of both genders for our analysis.

Lesions to the PFC in WT mice preferentially disrupt their ability to perform the DNMTS task (Kellendonk et al., 2006). Previous findings have demonstrated that NCAM-EC mice display fewer GABAergic inhibitory synapses throughout postnatal development of the PFC and, in particular, reduced numbers of perisomatic synapses of basket interneurons with pyramidal cells (Brennaman and Maness, 2008; Pillai-Nair et al., 2005). Perisomatic innervation of pyramidal neurons by basket interneurons is thought to promote synchronous firing of pyramidal neurons and regulate working memory (Klausberger et al., 2003; Sohal et al., 2009). Thus, the decrease in perisomatic innervation in the PFC of NCAM-EC mice might contribute to their decreased working memory ability in the DNMTS task. Intriguingly, social interactions of NCAM-EC mice were not different from those of WT mice, suggesting that the decreased perisomatic GABAergic innervation does not alter social behavioral responses.

Anxiety, olfaction, and locomotor activity in a novel environment were not contributing factors to this impairment, as these responses were normal. Previous studies of activity with the NCAM-EC transgenic mice showed increased locomotor activity in an open field over a $1 \mathrm{~h}$ monitoring period (Pillai-Nair et al., 2005). In the current study, no differences were 
seen in activity in a 5 min monitoring period, suggesting that longer periods of testing are required for increased locomotion to be observed.

Electrophysiological recordings in brain slices from NCAM-EC transgenic mice revealed decreased LTP and STP in the PFC. Normality of LTP and STP as well as normal basal transmission in the hippocampus suggest that the NCAM-EC protein affects two brain regions in distinct ways. Indeed, these functional distinctions are consistent with structural ones: decreased inhibitory synapses in the PFC and amygdala of NCAM-EC mice, and normal numbers of inhibitory synapses in the hippocampus (Pillai-Nair et al., 2005). NCAM-EC transgenic mice also show reduced numbers of apical dendritic spines on pyramidal neurons in the PFC, which could contribute to circuit alterations responsible for the abnormal behavioral responses and impaired synaptic plasticity responses observed here. The finding that overall basal transmission and excitatory paired-pulse facilitation were normal in NCAM-EC mice suggests that the reduced inhibitory and excitatory synaptic connectivity might in some way compensate each other in maintaining normal levels of neurotransmission.

NCAM-null mice lacking all isoforms of NCAM exhibit decreased hippocampal LTP (Kochlamazashvili et al., 2010; Muller et al., 1996), which is in contrast to our findings that NCAM-EC mice have normal hippocampal LTP. Furthermore, NCAM-null mice show increased anxiety (Stork et al., 1999), whereas NCAM-EC mice have normal levels of anxiety. These results suggest that altering the expression patterns of NCAM by increasing the amount of NCAM-EC or by decreasing the levels of NCAM in NCAM-null mice may differentially affect behavior and synaptic plasticity. The more "focused" phenotype of NCAM-EC mice as compared to NCAM-null mice is not surprising. Although NCAM-EC can compete with transmembrane NCAM, and thus inhibit NCAM-mediated transinteractions between neural cells (Brennaman and Maness, 2008), the concentration of NCAM-EC may be not sufficient to fully block NCAM-mediated adhesion and fully mimic NCAM-null mice. It is also possible that the region-specific effects of NCAM-EC are related to excessive or ectopic signaling via transmembrane forms of NCAM or heterophilic binding partners of NCAM in the PFC.

\section{Conclusions}

Our results demonstrate that NCAM-EC mice model not all aspects of schizophrenia, but specifically mimic impaired PFC functions in schizophrenia at the structural, physiological and behavioral levels. These mice, hence, provide a valuable tool for further mechanistic and pharmacological studies aiming in restoration of activity and plasticity in the PFC.

\section{Supplementary Material}

Refer to Web version on PubMed Central for supplementary material.

\section{Acknowledgments}

This work was supported by NIMH funding of University of North Carolina Silvio O. Conte Center for the Neuroscience of Mental Disorders (MH064065; P.F.M.), 2009 NARSAD William Risser Charitable Trust Investigator/NARSAD Young Investigator Award (L.H.B.), NICHD funding of the Carolina Institute for Developmental Disabilities (P30 HD03110, Joe Piven), by Deutsche Forschungsgemeinschaft (Di 702/4-3 A.D.) and Italian Institute of Technology (A.D.) and by the University Medical Center Hamburg-Eppendorf (M.S.).

\section{References}

Al-Uzri MM, et al. Measuring memory impairment in community-based patients with schizophrenia. Case-control study. Br J Psychiatry. 2006; 189:132-6. [PubMed: 16880482] 
Anderson SW, et al. Consistency of neuropsychological outcome following damage to prefrontal cortex in the first years of life. J Clin Exp Neuropsychol. 2009; 31:170-9. [PubMed: 19051128]

Arikkath J, Reichardt LF. Cadherins and catenins at synapses: roles in synaptogenesis and synaptic plasticity. Trends Neurosci. 2008; 31:487-94. [PubMed: 18684518]

Becker CG, et al. The polysialic acid modification of the neural cell adhesion molecule is involved in spatial learning and hippocampal long-term potentiation. J Neurosci Res. 1996; 45:143-152. [PubMed: 8843031]

Bonfanti L. PSA-NCAM in mammalian structural plasticity and neurogenesis. Prog Neurobiol. 2006; 80:129-64. [PubMed: 17029752]

Brady AM, et al. Selective deficits in spatial working memory in the neonatal ventral hippocampal lesion rat model of schizophrenia. Neuropharmacology. 2010; 59:605-11. [PubMed: 20732335]

Bramham J, et al. Social and emotional functioning following bilateral and unilateral neurosurgical prefrontal cortex lesions. J Neuropsychol. 2009; 3:125-43. [PubMed: 19338721]

Brennaman LH, Maness PF. Developmental regulation of GABAergic interneuron branching and synaptic development in the prefrontal cortex by soluble neural cell adhesion molecule. Mol Cell Neurosci. 2008; 37:781-93. [PubMed: 18289872]

Brennaman LH, Maness PF. NCAM in neuropsychiatric and neurodegenerative disorders. Adv Exp Med Biol. 2010; 663:299-317. [PubMed: 20017030]

Cassens $\mathrm{C}$, et al. Binding of the receptor tyrosine kinase TrkB to the neural cell adhesion molecule NCAM regulates phosphorylation of NCAM and NCAM-dependent neurite outgrowth. J Biol Chem. 2010

Clapcote SJ, et al. Behavioral phenotypes of Disc1 missense mutations in mice. Neuron. 2007; 54:387-402. [PubMed: 17481393]

Cox ET, et al. Developmental regulation of neural cell adhesion molecule in human prefrontal cortex. Neuroscience. 2009; 162:96-105. [PubMed: 19393299]

Cremer $\mathrm{H}$, et al. Inactivation of the N-CAM gene in mice results in size reduction of the olfactory bulb and deficits in spatial learning. Nature. 1994; 367:455-459. [PubMed: 8107803]

Dalva MB, et al. Cell adhesion molecules: signalling functions at the synapse. Nat Rev Neurosci. 2007; 8:206-20. [PubMed: 17299456]

Dityatev A, et al. Modulation of synaptic transmission and plasticity by cell adhesion and repulsion molecules. Neuron Glia Biol. 2008; 4:197-209. [PubMed: 19674506]

Duffy. neuroscience. 2010.

Eckhardt M, et al. Mice deficient in the polysialyltransferase ST8SiaIV/PST-1 allow discrimination of the roles of neural cell adhesion molecule protein and polysialic acid in neural development and synaptic plasticity. J Neurosci. 2000; 20:5234-5244. [PubMed: 10884307]

Finn AS, et al. Longitudinal evidence for functional specialization of the neural circuit supporting working memory in the human brain. J Neurosci. 2010; 30:11062-7. [PubMed: 20720113]

Gehring WJ, Knight RT. Lateral prefrontal damage affects processing selection but not attention switching. Brain Res Cogn Brain Res. 2002; 13:267-79. [PubMed: 11958971]

Gottmann K. Transsynaptic modulation of the synaptic vesicle cycle by cell-adhesion molecules. J Neurosci Res. 2008; 86:223-32. [PubMed: 17787017]

Hafner H, an der Heiden W. Epidemiology of schizophrenia. Can J Psychiatry. 1997; 42:139-51. [PubMed: 9067063]

Hafner H, et al. Causes and consequences of the gender difference in age at onset of schizophrenia. Schizophr Bull. 1998; 24:99-113. [PubMed: 9502549]

Hildebrandt H, et al. Dissecting polysialic acid and NCAM functions in brain development. J Neurochem. 2007; 103(Suppl 1):56-64. [PubMed: 17986140]

Hinkle CL, et al. Metalloprotease-induced ectodomain shedding of neural cell adhesion molecule (NCAM). Journal of Neurobiology. 2006; 66:1378-95. [PubMed: 16967505]

Hovatta I, et al. Schizophrenia in the genetic isolate of Finland. Am J Med Genet. 1997; 74:353-60. [PubMed: 9259368] 
Huang YY, et al. Genetic evidence for the bidirectional modulation of synaptic plasticity in the prefrontal cortex by D1 receptors. Proc Natl Acad Sci U S A. 2004; 101:3236-41. [PubMed: 14981263]

Jurgenson M, et al. Behavioral profile of mice with impaired cognition in the elevated plus-maze due to a deficiency in neural cell adhesion molecule. Pharmacol Biochem Behav. 2010; 96:461-468. [PubMed: 20624419]

Kadmon G, et al. The neural cell adhesion molecule N-CAM enhances L1-dependent cell-cell interactions. J Cell Biol. 1990; 110:193-208. [PubMed: 2295682]

Kalus I, et al. Proteolytic cleavage of the neural cell adhesion molecule by ADAM17/TACE is involved in neurite outgrowth. J Neurochem. 2006; 98:78-88. [PubMed: 16805798]

Kellendonk C, et al. Modeling cognitive endophenotypes of schizophrenia in mice. Trends Neurosci. 2009; 32:347-58. [PubMed: 19409625]

Kellendonk C, et al. Transient and selective overexpression of dopamine D2 receptors in the striatum causes persistent abnormalities in prefrontal cortex functioning. Neuron. 2006; 49:603-15. [PubMed: 16476668]

Klausberger T, et al. Brain-state- and cell-type-specific firing of hippocampal interneurons in vivo. Nature. 2003; 421:844-8. [PubMed: 12594513]

Kochlamazashvili G, et al. Neural cell adhesion molecule-associated polysialic acid regulates synaptic plasticity and learning by restraining the signaling through GluN2B-containing NMDA receptors. J Neurosci. 2010; 30:4171-83. [PubMed: 20237287]

Lüthi A, et al. Hippocampal long-term potentiation and neural cell adhesion molecules L1 and NCAM. Nature. 1994; 372:777-779. [PubMed: 7997264]

Manes F, et al. Decision-making processes following damage to the prefrontal cortex. Brain. 2002; 125:624-39. [PubMed: 11872618]

Mohamed S, et al. Generalized cognitive deficits in schizophrenia: a study of first-episode patients. Arch Gen Psychiatry. 1999; 56:749-54. [PubMed: 10435610]

Moy SS, et al. Sociability and preference for social novelty in five inbred strains: an approach to assess autistic-like behavior in mice. Genes Brain Behav. 2004; 3:287-302. [PubMed: 15344922]

Muller D, et al. PSA-NCAM is required for activity-induced synaptic plasticity. Neuron. 1996; 17:413-422. [PubMed: 8816705]

Myles-Worsley M, et al. Genetic epidemiological study of schizophrenia in Palau, Micronesia: prevalence and familiality. Am J Med Genet. 1999; 88:4-10. [PubMed: 10050960]

Nadler JJ, et al. Automated apparatus for quantitation of social approach behaviors in mice. Genes Brain Behav. 2004; 3:303-14. [PubMed: 15344923]

Nielsen J, et al. Role of glial cell line-derived neurotrophic factor (GDNF)-neural cell adhesion molecule (NCAM) interactions in induction of neurite outgrowth and identification of a binding site for NCAM in the heel region of GDNF. J Neurosci. 2009; 29:11360-76. [PubMed: 19741142]

Niethammer P, et al. Cosignaling of NCAM via lipid rafts and the FGF receptor is required for neuritogenesis. J Cell Biol. 2002; 157:521-532. [PubMed: 11980923]

Paratcha G, et al. The neural cell adhesion molecule NCAM is an alternative signaling receptor for GDNF family ligands. Cell. 2003; 113:867-879. [PubMed: 12837245]

Pillai-Nair N, et al. Neural cell adhesion molecule-secreting transgenic mice display abnormalities in GABAergic interneurons and alterations in behavior. J Neurosci. 2005; 25:4659-4671. [PubMed: 15872114]

Polo-Parada L, et al. Alterations in transmission, vesicle dynamics, and transmitter release machinery at NCAM-deficient neuromuscular junctions. Neuron. 2001; 32:815-828. [PubMed: 11738028]

Saha S, Chant D, Welham J, McGrath J. A systematic review of the prevalence of schizophrenia. PLoS Med. 2005; 2:e141. [PubMed: 15916472]

Schachner M. Neural recognition molecules and synaptic plasticity. Curr Opin Cell Biol. 1997; 9:62734. [PubMed: 9330865]

Senkov O, et al. Polysialylated neural cell adhesion molecule is involved in induction of long-term potentiation and memory acquisition and consolidation in a fear-conditioning paradigm. $\mathrm{J}$ Neurosci. 2006; 26:10888-109898. [PubMed: 17050727] 
Sohal VS, et al. Parvalbumin neurons and gamma rhythms enhance cortical circuit performance. Nature. 2009; 459:698-702. [PubMed: 19396159]

Stark KL, et al. Altered brain microRNA biogenesis contributes to phenotypic deficits in a 22q11deletion mouse model. Nat Genet. 2008; 40:751-60. [PubMed: 18469815]

Stoenica L, et al. In vivo synaptic plasticity in the dentate gyrus of mice deficient in the neural cell adhesion molecule NCAM or its polysialic acid. Eur J Neurosci. 2006; 23:2255-64. [PubMed: 16706834]

Stork $\mathrm{O}$, et al. Increased intermale aggression and neuroendocrine response in mice deficient for the neural cell adhesion molecules. Eur J Neurosci. 1997; 9:424-434. [PubMed: 9104585]

Stork O, et al. Recovery of emotional behaviour in neural cell adhesion molecule (NCAM) null mutant mice through transgenic expression of NCAM180. Eur J Neurosci. 2000; 12:3291-3306. [PubMed: 10998113]

Stork O, et al. Anxiety and increased 5-HT1A receptor response in NCAM null mutant mice. J Neurobiol. 1999; 40:343-355. [PubMed: 10440734]

Storms SD, Rutishauser U. A role for polysialic acid in neural cell adhesion molecule heterophilic binding to proteoglycans. J Biol Chem. 1998; 273:27124-27129. [PubMed: 9765230]

Vawter MP. Dysregulation of the neural cell adhesion molecule and neuropsychiatric disorders. Eur J Pharmacol. 2000; 405:385-395. [PubMed: 11033343] 
Research Highlights

Increase in a soluble form of NCAM is commonly found in schizophrenic brains

Here, we studied NCAM-EC mice overexpressing this soluble form of NCAM

NCAM-EC mice exhibit impaired working memory in a delayed non-match-to-sample task

NCAM-EC mice have impaired long- and short-term potentiation in the prefrontal cortex

Thus, NCAM-EC mice is a model of impaired prefrontal functions in schizophrenia 

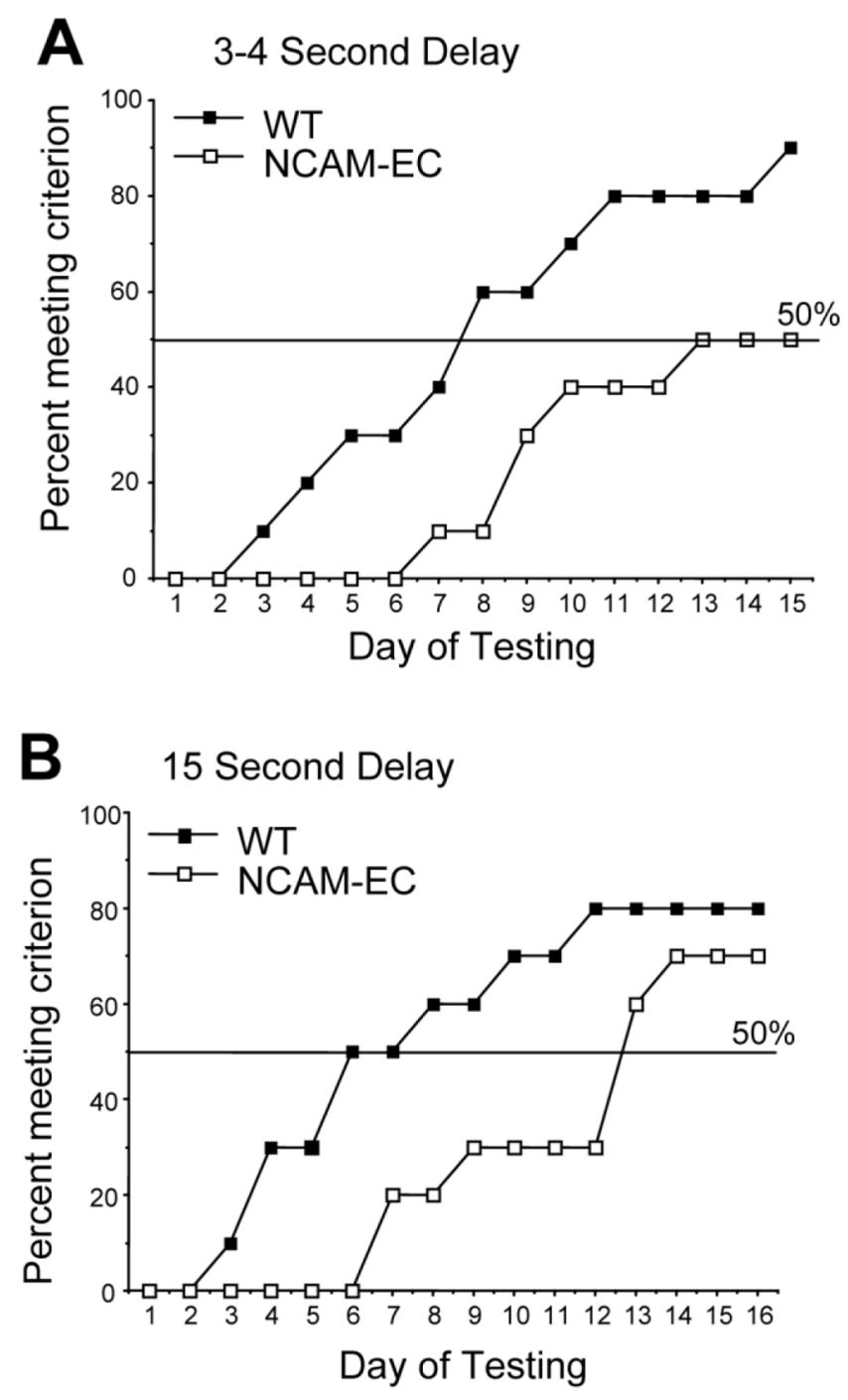

Figure 1. NCAM-EC mice exhibit impaired working memory in the delayed non-match-tosample task

Mice ( $n=10$ of each genotype) were given 4 trials per day, with each trial consisting of a free choice and a forced choice run separated by a short delay. Criterion for learning was set at 11 out of 12 correct choices in the forced choice portion over 3 consecutive days. Days 1 and 2 were training days. (A) Delay of 3-4 s between free choice and forced choice. Ninety percent of WT mice and 50\% of NCAM-EC transgenic mice met criterion by 15 days of testing. (B) Delay of $15 \mathrm{~s}$ between free choice and forced choice. Eighty percent of WT mice and $70 \%$ of NCAM-EC transgenic mice met criterion by 16 days of testing. 

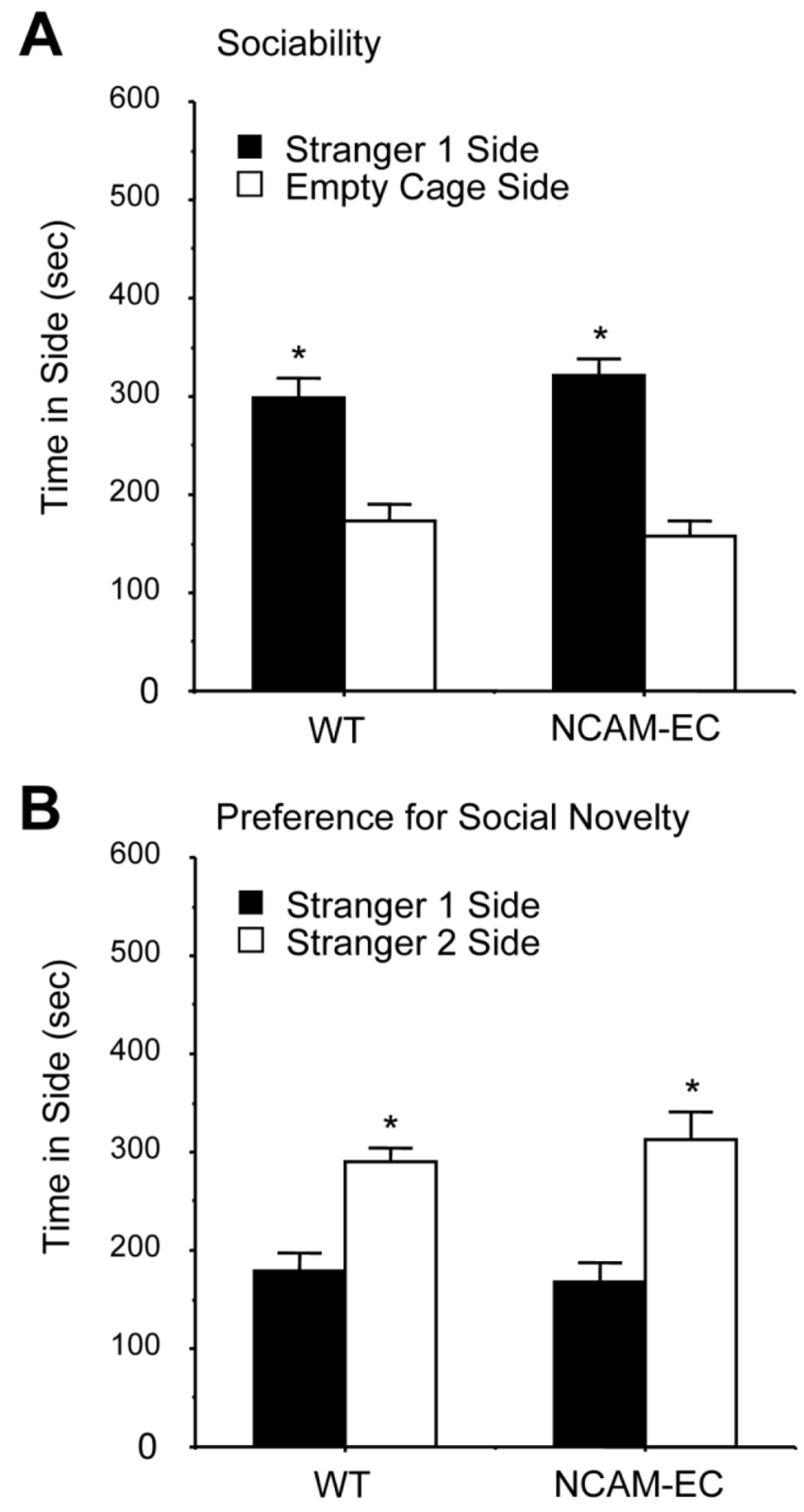

Figure 2. NCAM-EC mice exhibit normal sociability and preference for social novelty Time spent in each of the side chambers during the test for sociability (A) and social novelty (B). In the social novelty test, Stranger 1 had been previously investigated during the sociability assay, while Stranger 2 was a newly-introduced unfamiliar mouse. Data shown are mean + SEM for each group $(n=10 \mathrm{WT}, 9$ NCAM-EC) for a 10 min test. * $\mathrm{p}<0.05$, within-group comparison between stranger 1 side and opposite side. 
A

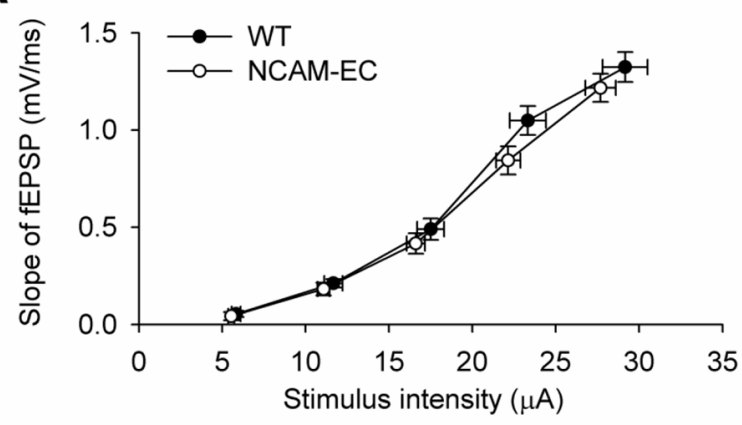

B

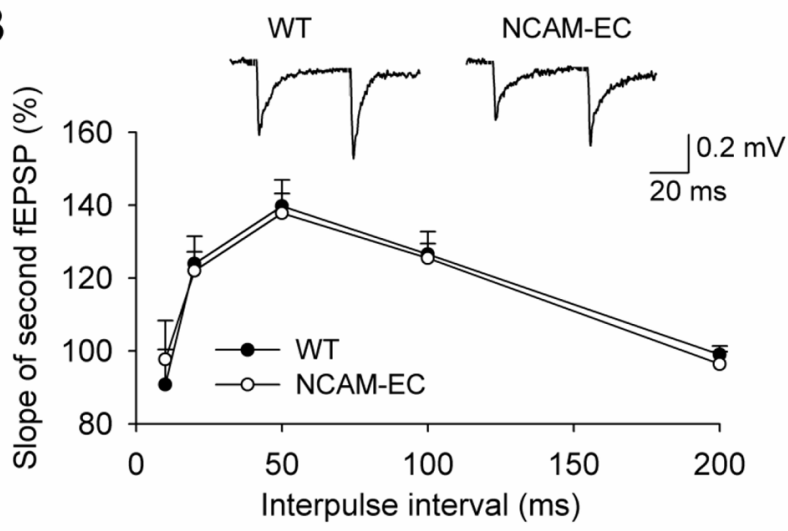

C

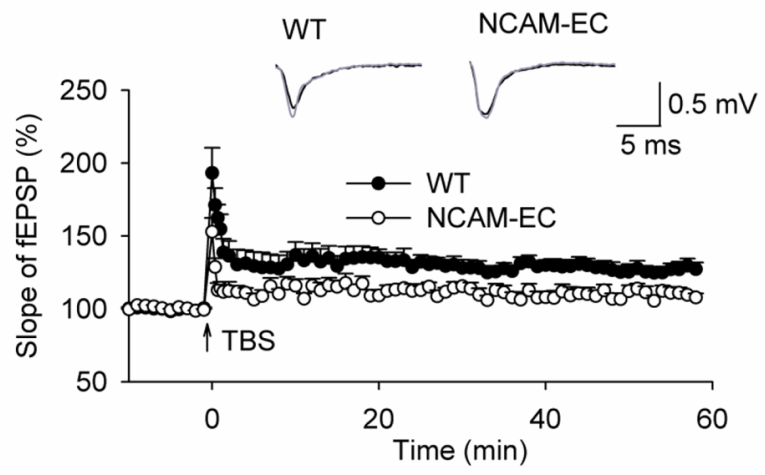

Figure 3. Impaired synaptic plasticity in PFC slices from NCAM-EC mice

(A) Relationships between stimulus intensity and slope of fEPSPs. No effect of NCAM-EC expression was detected on the slope $(\mathrm{F}(1,23)=2.061, \mathrm{p}=0.165)$ or stimulus intensity $(\mathrm{F}(1,23)=0.709, \mathrm{p}=0.408)$. (B) Examples of paired-pulse facilitation in NCAM-EC and WT mice at $50 \mathrm{~ms}$ interpulse interval (insert) and relationships between interpulse interval and paired-pulse facilitation, measured as a ratio between the slopes of second and first responses expressed in \%. No effect of NCAM-EC expression was detected $(\mathrm{F}(1,23)=0.0004, \mathrm{p}=0.984)$. (C) Impaired STP and LTP induced by theta-burst stimulation (TBS) in the PFC in NCAM-EC mice in comparison to WT mice (see the Results). The mean slope of fEPSPs recorded 10 min before TBS was taken as $100 \%$ and arrows indicate delivery of TBS, as indicated on panels. Traces in insets show the averages of 30 fEPSPs recorded 10 min before or 50-60 min after induction of LTP. Data represent means + SEM from 9-12 slices recorded in 5 WT mice and 11-13 slices from 6 NCAM-EC mice. 
A
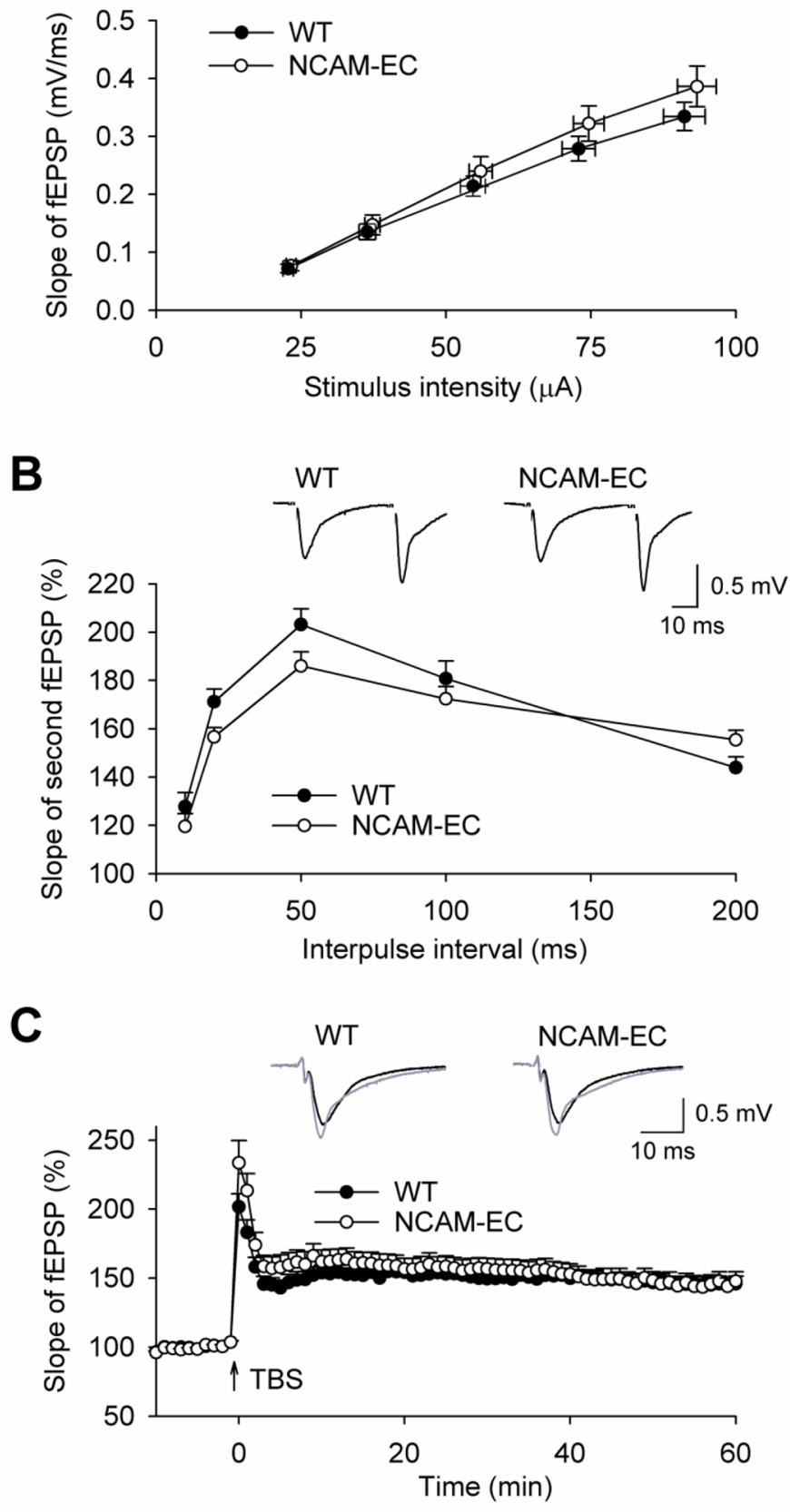

Figure 4. Normal levels of basal synaptic transmission, short- and long-term synaptic plasticity in hippocampal slices from NCAM-EC mice

(A) Relationships between stimulus intensity and slope of fEPSPs. No effect of NCAM-EC expression was detected by ANOVA with repeated measures for the slope $(F(1,20)=0.967$, $\mathrm{p}=0.337)$ or stimulus intensities $(\mathrm{F}(1,20=0.259, \mathrm{p}=0.616)$. (B) Examples of paired-pulse facilitation in NCAM-EC and WT mice at $50 \mathrm{~ms}$ interpulse interval (inserts) and relationships between interpulse interval and paired-pulse facilitation, measured as a ratio between the slopes of second and first responses expressed in \%. For short intervals (10 and $20 \mathrm{~ms}$ ), the first fEPSP was digitally subtracted before measurements of the second fEPSP. No effect of genotype was detected by ANOVA $(F(1,14)=1.291, p=0.275)$. (C) Normal 
STP and LTP induced by theta-burst stimulation (TBS) in the CA1 region of NCAM-EC mice (see the Results). Presentation of data is as in Fig. 3. Data represent means + SEM from 9-13 slices recorded in 4 WT mice and 7-9 slices from 4 NCAM-EC mice. 
Table 1

Anxiety-like behavior on an elevated plus maze, and olfactory ability in a buried-food test.

\begin{tabular}{lll}
\hline & WT (n=10) & NCAM-EC (n=9) \\
\hline Open field & & \\
$\quad$ Total distance traveled (cm) & $902 \pm 124$ & $1010 \pm 113$ \\
$\quad$ Rearing movements & $26 \pm 4$ & $27 \pm 3$ \\
$\quad$ Time spent in the center region (sec) & $18 \pm 4$ & $19 \pm 4$ \\
Elevated plus maze & & \\
$\quad$ Percent open arm time & $10 \pm 1$ & $9 \pm 2$ \\
$\quad \begin{array}{l}\text { Percent open arm entries } \\
\text { Total number of entries }\end{array}$ & $21 \pm 3$ & $16 \pm 3$ \\
Olfactory test (without food deprivation) & & $20 \pm 2$ \\
$\quad$ Latency to find food (sec) & $671 \pm 101$ & $455 \pm 124$ \\
$\quad$ Percent of group finding food & $40 \%$ & $67 \%$ \\
Olfactory test (with food deprivation) & & \\
$\quad$ Latency to find food (sec) & $279 \pm 102$ & $363 \pm 113$ \\
$\quad$ Percent of group finding food & $90 \%$ & $89 \%$ \\
\hline
\end{tabular}

Data shown are means \pm SEM. Results from the open field, elevated plus maze, and olfactory test (latency measures) were analyzed using t-tests. 